\title{
A New Backscattering Model for Nano-MOSFET Compact Modeling
}

\author{
E. Fuchs ${ }^{1,2}$, P. Dollfus ${ }^{2}$, G. Lecarval ${ }^{3}$, E. Robilliart ${ }^{1}$, \\ S. Barraud ${ }^{3}$, D. Villanueva ${ }^{4}$, H. Jaouen ${ }^{1}$. \\ ${ }^{1}$ STMicroelectronics, F-38926 Crolles, France,emmanuel.fuchs@st.com. \\ ph: +33 $476925956,{ }^{2}$ IEF, CNRS-UPS Orsay, France, ${ }^{3}$ CEA-SRD-DTS-LSPC, \\ Grenoble, France, ${ }^{4}$ Philips Semiconductors, Crolles, France
}

\begin{abstract}
For fast computation of drain current in Nano-MOSFET, we have developed a new backscattering model based on the accurate determination of ballistic and backscattering probabilities along the channel. The main elements of this model are deduced from careful analysis of transport in devices using Monte Carlo simulation. The backscattering coefficient is in very good agreement with the results of Monte Carlo spectroscopy for MOS transistors and $\mathrm{N}+/ \mathrm{N} / \mathrm{N}+$ diodes.
\end{abstract}

\section{Introduction}

Recent works showed that nano-MOSFETs operate close to the ballistic limits [1]. In this regime, an accurate estimation of the backscattering coefficient $R_{C}$ is needed for a realistic $I_{D}$ calculation using the simple expression $[2,3]$

$$
I_{D}=C_{o x} W\left(V_{G}-V_{t h}\right) v_{T} \frac{1-R_{C}}{1+R_{C}}\left[\frac{1-e^{-q V D s} / k T}{1+\frac{1-R_{C}}{1+R_{C}} e^{-q V} / k T}\right]
$$

In this work, we propose a physically improved backscattering model based on energy-dependent scattering rates. It is simple enough to be used in predictive compact modeling, which is very useful in a R\&D context, and it is validated via careful comparison with Monte Carlo simulation for three MOSFETs designed from ITRS specifications (Fig. 1) and for $\mathrm{N}+/ \mathrm{N} / \mathrm{N}+$ diodes.

\section{Backscattering model}

The backscattering coefficient $R_{C}$ is usually determined from the mean free path $\lambda$ and the critical distance $l$ over which the channel potential drops by $k T / q$ (Fig.2)[2]; i.e. $R_{c}=l /(\lambda+l)$. This approach postulates that most carriers that contribute to the backscattered flux scatter within the so-called " $k T$-layer". But recent works suggest that in very short MOSFETs the entire channel or a more significant part of the channel participates in the backscattering [4,5]. Moreover, the determination of $\lambda=v_{T} \times \tau$ (Fig.2) is usually obtained from a single effective mass thermal velocity $v_{T}$ 
and a near-equilibrium relaxation time $\tau$, which is questionable in the case of highenergy backscattered electrons.

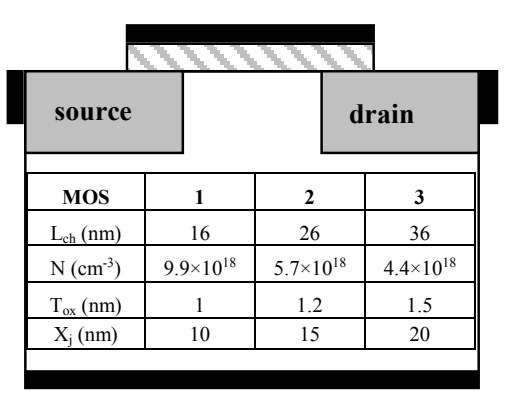

Figure 1: Description of 3 bulk MOSFETs designed from ITRS specifications

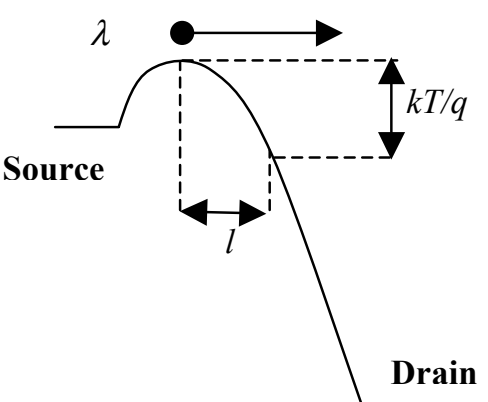

Figure 2: Schematic conduction band energy vs. position for a MOSFET under high gate and drain bias.

Preliminary Monte Carlo simulation has been performed to get information on transport and scattering in the channel. $R_{c}$ can be obtained from the velocity spectrum at the top of the barrier (Fig.3) as the ratio of negative flux to positive flux.

The velocity spectrum extracted in the middle of the channel (Fig.4) presents a negative velocity part whose a fraction contributes to the backscattered flux. The evaluation of this fraction at each position of the channel requires us to study separately the contribution of populations with either a transverse mass $\left(m_{t}\right)$ or a longitudinal mass $\left(m_{l}\right)$. The ballistic part of these populations is clearly evidenced by two peaks in Fig.4. The results suggest that the correct estimation of $R_{C}$ should derive from the integration of backscattering probabilities over the entire channel length.

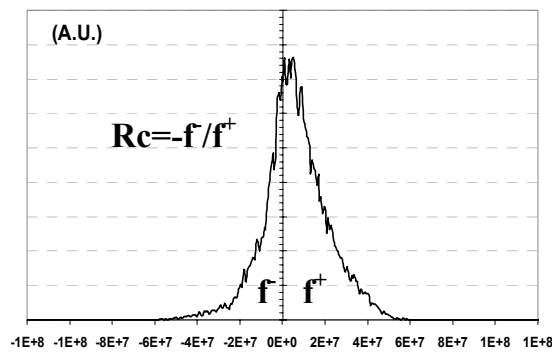

Velocity $(\mathrm{cm} / \mathrm{s})$

Figure 3: Velocity spectrum at the top of the barrier for MOS2 at $V_{D S}=0.8 \mathrm{~V}$

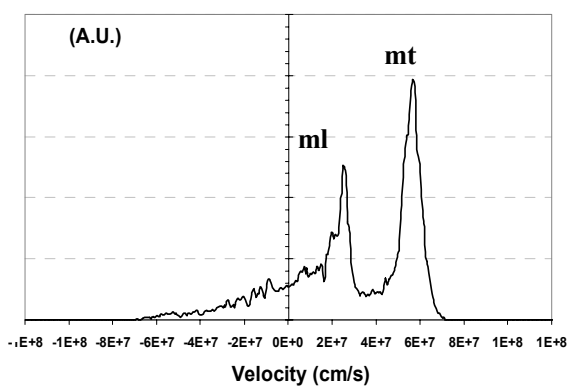

Figure 4: Velocity spectrum at the middle of the channel of MOS2 at $V_{G S}=V_{D S}=0.8 \mathrm{~V}$.

In our model, we thus consider two thermal velocities, i.e. $v_{T}=1.2 \times 10^{5} \mathrm{~m} / \mathrm{s}$ for "transverse" electrons and $5.6 \times 10^{4} \mathrm{~m} / \mathrm{s}$ for "longitudinal" electrons. Assuming a parabolic channel potential at given drain bias, we calculate the ballistic velocity $v$ bal and the ballistic energy for each type of carriers as a function of the position $x$ in the 
channel. From this energy we determine the total scattering rate of ballistic carriers $1 / \tau_{b a l}(x)$ using the same scattering rate expressions as used for Monte Carlo simulation. A "dynamic free path" $D f p$ is then calculated as $D f p(x)=v_{b a l}(x) \times \tau_{b a l}(x)$. For a ballistic carrier at $x$, this quantity represents the average distance at which a scattering event should be experienced. As shown in Fig.5, Dfp increases along the channel at the source-end due to the velocity increase, and decreases at the drain-end because of the strong increase of phonon emission rate at high energy. The fraction $N_{b a l}(x+d x)$ of ballistic electrons at $x+d x$ can be deduced from $N_{b a l}(x)$ as $N_{b a l}(x+d x)=N_{b a l}(x) \times \exp (-d x / D f p(x))$. This function $N_{b a l}(x)$ is plotted in Fig.6 for diodes with two doping levels in the $\mathrm{N}$ central region and compares very well with Monte Carlo results.

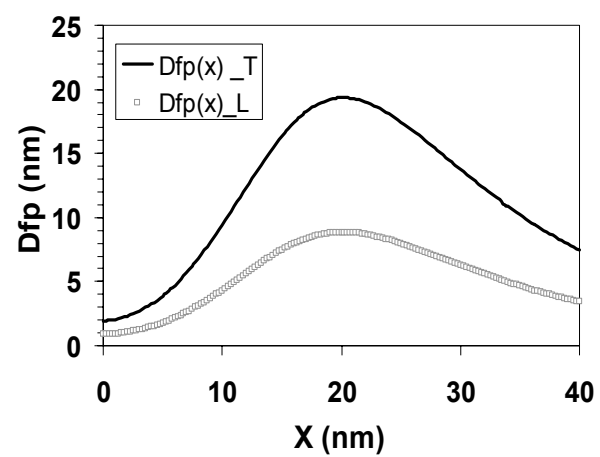

Figure 5: Dfp_T and Dfp_L versus the distance along the channel for MOS2 at $V_{D S}=0.8 \mathrm{~V}$

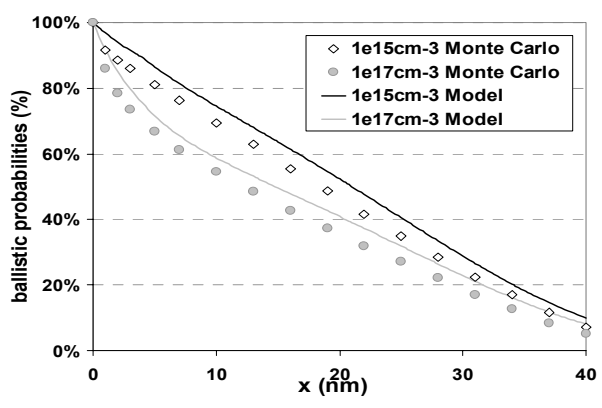

Figure 6: Ballistic probability $N_{b a l}(x)$ versus the distance along the channel for a diode $L_{c h}=40 \mathrm{~nm}$ at $V_{D S}=1 \mathrm{~V}$

From the probability $N_{b a l}(x)$ we can deduce the probability $N_{s c a t}(x)$ for an electron to have its first scattering event at position $x$. For a carrier having just scattered at $\mathrm{x}$ we calculate the probability to be backscattered. Taking into account the possible change in energy after scattering at $x$, we calculate the probability $P(x)$ that the carrier reenters the source region by determining the transmission coefficient to the source $T_{S}(x)$ under a repulsive electric field and the coefficient transmission to the drain $T_{D}(x)$ under a attractive electric field [6]. The probability $P(x)$ is equal to $T_{S}(x) /\left(T_{S}(x)+T_{D}(x)\right)$. By integrating these different contributions over the channel length, we obtain the fraction of backscattering as a function of $x$ for both types of carriers, as illustrated in Fig.7. By weighting these quantities by the carrier distribution in "transverse" and "longitudinal" valleys, the final $R_{C}$ is determined as the value of the fraction of backscattering at $x=L_{c h}$. As illustrated in Fig.8, our model gives accurate $R_{C}$ values for several MOSFETs and $\mathrm{N}+\mathrm{NN}+$ diodes in comparison with Monte Carlo results (error less than 5\%) and [2]. We have thus developed an efficient and rigorous backscattering model. This model takes into account the non-equilibrium transport and inspects the different possible contributions of each type of carrier all along the channel. 


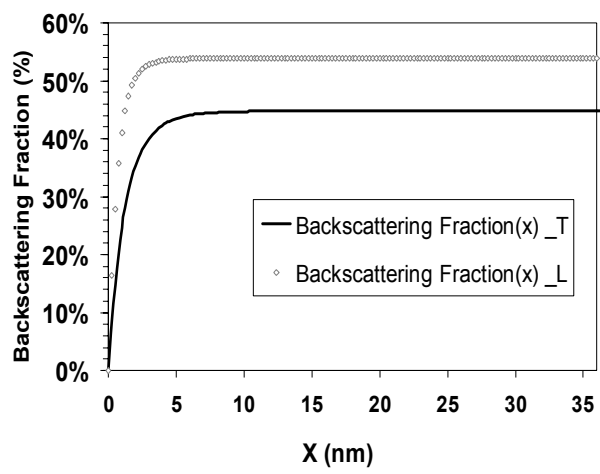

Figure 7 : Fraction of backscattering versus the distance along the channel for MOS1 at $V_{D S}=0.8 \mathrm{~V}$

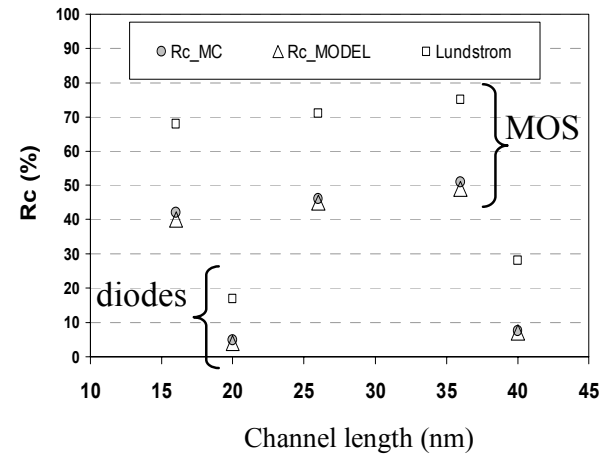

Figure 8: Comparison of $R_{c}$ models and Monte Carlo results for 3 MOSFETs at $V_{D S}=0.8 \mathrm{~V}$ and 2 $\mathrm{N}+/ \mathrm{N} / \mathrm{N}+\operatorname{diodes}\left(5.10^{19} \mathrm{~cm}^{-3} /\right.$ $\left.10^{15} \mathrm{~cm}^{-3} / 5.10^{19} \mathrm{~cm}^{-3}\right)$ at $V_{D S}=1 \mathrm{~V}$

\section{Compact Modeling}

This backscattering model allows us to calculate the resulting injection velocity with $v_{i n j}=v_{T}\left(1-R_{C} / 1+R_{C}\right)$. Then, after the calculation of the charge at the top of the barrier, the drain current may be deduced from Eq.(1). This model is the foundation of a new compact modeling approach based on microscopic observations. Its implementation in a full compact modeling environment is currently underway.

\section{Acknowledgements to ARTEMIS European project funding}

\section{References}

[1] K. Natori, "Ballistic MOSFET Reproduces Current-Voltage Characteristics of an Experimental Device", IEEE Electron Dev. Lett., vol. 23, pp. 655-657, 2002

[2] M.S. Lundstrom, "Elementary scattering theory of the Si MOSFET", IEEE Electron Dev. Lett., vol. 18, pp. 361-363, 1997.

[3] M.S. Lundstrom, "A Landauer Approach to Nanoscale MOSFETs", Journal of Computational Electronics, vol. 1, pp. 481-489, 2002.

[4] A. Svizhenko and M.P. Anantram, "Role of scattering in Nanotransistor", IEEE Trans. Electron Dev., vol. 50, pp. 1459-1466, 2003.

[5] J. Saint Martin, A. Bournel, P. Dollfus, "On the ballistic transport in nanometer-scaled double-gate MOSFET", to be published in IEEE Trans. Electron Dev (July 2004).

[6] M. Lundstrom, in Fundamentals of carrier transport, Cambridge, 2000. 